hep-ph@xxx/9203221

UCLA $/ 92 / \mathrm{TEP} / 9$

MCGILL/92-11

\title{
Improved Heavy Quark Effective Theory Currents
}

\author{
Oscar F. Hernández \\ Department of Physics \\ McGill University \\ Ernest Rutherford Physics Building \\ Montréal, Qué., Canada H3A 2T8
}

Brian R. Hill

Department of Physics

University of California

Los Angeles, CA 90024

\begin{abstract}
It is hoped that the accuracy of a variety of lattice calculations will be improved by perturbatively eliminating effects proportional to the lattice spacing. In this paper, we apply this improvement program to the heavy quark effective theory currents which cause a heavy quark to decay to a light quark, and renormalize the resulting operators to order $\alpha_{S}$. We find a small decrease in the amount that the operator needs to be renormalized, relative to the unimproved case.
\end{abstract}




\section{Introduction}

It is hoped that the accuracy of a variety of lattice calculations will be improved by the elimination of effects proportional to powers of the lattice spacing, $a$ [1][2]

. This improvement program can be implemented order by order in $a$ and the strong coupling $g^{2}$. Calculationally, it is relatively easy to perform the leading order in either of these expansions. For matrix elements of vector currents, both types of leading order corrections are thought to be in the 20 to $30 \%$ range [3], so it is at the same time important to include them, and reasonable to stop at this order.

Much of the application of the improvement program to hadronic matrix elements has been to the order $a$ improvement of the Wilson fermion action and operators [4]-[6]. Alternative methods for measuring matrix elements involving quarks that are heavy relative to the QCD scale have been proposed [7][8]. The action and operators in this method can be thought of as discretizations of the heavy quark effective field theory action and operators [8][9]. In this paper, we study the application of the improvement program to this action and the currents which cause a heavy quark to decay to a light quark.

The outline of the paper is as follows. In the next section, we discuss improvement of the heavy quark action, and in section three, we discuss improvement of the aforementioned currents. As is generally the case, the improved lattice currents need to be related to their continuum counterparts. This is done perturbatively in $g^{2}$ where the scale is set by the lattice spacing. Although quite different in motivation, this perturbative calculation is similar to the renormalization of other discretizations of the heavy quark effective theory currents performed in reference [10]. That calculation and the present one rely on the framework and results of reference [11], which are summarized in section four before the new perturbative results are presented. In section five we give our conclusions.

\section{Improvement of the Heavy Quark Action}

In this section, we argue that under two criteria of improvement, there is no need to modify the heavy quark action. The arguments are tree level, but this is adequate for the leading order improvement discussed in the introduction. 
Roughly, the on-shell improvement condition formulated by Lüscher and Weisz [2] is that spectral quantities such as the location of single-particle poles should not be corrected by terms proportional to powers of the lattice spacing. Examining the momentum space propagator for the heavy quark action in the continuum [9],

$$
\frac{1}{p_{0}+i \epsilon}
$$

and comparing it with its lattice counterpart [11],

$$
\frac{1}{-i\left(e^{i p_{0} a}-1\right) / a+i \epsilon},
$$

we see that although the propagators differ at order $a$, the pole is located at $p_{0}=0$ to all orders in $a$. We immediately conclude that the action has no need of improvement under the on-shell improvement condition, to all orders in $a$ at tree level.

A second argument yielding this conclusion is to compare the heavy quark propagator in position space in an external gauge field on the lattice to the same thing in the continuum. ${ }^{\dagger}$ In the continuum, the propagator from $x$ to $y$ is [12],

$$
-i \delta^{3}(\mathbf{y}-\mathbf{x}) \theta\left(y_{0}-x_{0}\right) P \exp -i g \int_{x_{0}}^{y_{0}} d t A_{0}(t, \mathbf{x}) .
$$

The discretization of the action with a nearest neighbor, one-sided time derivative follows from this propagator [11]. On the lattice, the heavy quark propagator from $m$ to $n$ in a background field is [7][8],

$$
\frac{-i}{a^{3}} \delta_{\mathbf{n m}} \theta\left(n_{0}-m_{0}\right) U_{0}(n-\hat{\mathbf{0}})^{\dagger} U_{0}(n-2 \hat{\mathbf{0}})^{\dagger} \cdots U_{0}(m)^{\dagger}
$$

In this expression, $\delta_{\mathbf{n m}}$ is the three-dimensional Kronecker $\delta$-function, $\theta\left(n_{0}-m_{0}\right)$ is 1 if $n_{0} \geq m_{0}$ and 0 otherwise, and $U_{0}(m)$ is the lattice gauge link in the time direction at site $m$.

If the correspondence between continuum and lattice gauge fields is [2],

$$
U_{0}(n)=P \exp i g a \int_{0}^{1} d t A_{0}\left(\left(n_{0}+1-t\right) a, \mathbf{n} a\right)
$$

then the lattice propagator perfectly reproduces its continuum counterpart. If one modifies the heavy quark action (by choosing any other discretization of the

\footnotetext{
$\dagger$ We thank Estia Eichten for discussions leading to this argument.
} 
time derivative) the position space lattice propagator (2.4) is multiplied by a $c$ number factor depending only on $n_{0}-m_{0}$ but is otherwise unchanged (as long as the discretization does not include spatial links). With the correspondence (2.5), there is clearly no closer approximation to the continuum propagator (2.3) than the propagator (2.4) obtained from the action currently in use.

\section{Improved Heavy-Light Currents}

Since the lattice heavy quark action is not in need of improvement, we review the situation for the light quark action. Expressions for improved lattice heavy-light currents quickly follow from this discussion.

The improved Wilson fermion action proposed by Sheikholeslami and Wohlert [6] is obtained from an improved action with next-to-nearest neighbor couplings [4] by a change of variables. The resulting action is the Wilson action plus an additional piece,

$$
\Delta S_{I}=-i a^{4} \frac{a r}{4} \sum_{n \mu \nu} \bar{q}(n) \sigma_{\mu \nu} g P_{\mu \nu}(n) q(n)
$$

$P_{\mu \nu}(n)$ is the sum of plaquettes defined in reference [5] and goes to $F_{\mu \nu}(n)$ in the continuum limit, while $\sigma_{\mu \nu}=\left[\gamma_{\mu}, \gamma_{\nu}\right] / 2$. The action has the advantage that it only contains nearest neighbor couplings. One can most easily obtain improved operators for use with this action by starting with local bilinears and making the replacement [3][6],

$$
q(n) \rightarrow q(n)-\frac{r}{2} \sum_{\mu} \gamma_{\mu}\left[U_{\mu}(x) q(n+\mu)-U_{\mu}(n-\hat{\mu})^{\dagger} q(n-\hat{\mu})\right]
$$

A similar replacement is made for $\bar{q}(n)$.

Actually, a two-parameter family of transformations, all of which yield operators improved to order $a$, can be obtained by using the equations of motion for the light and heavy quark. The effect of applying the equation of motion to the light quark field has been discussed in reference [5] and the effect of applying the equation of motion to the heavy quark field has been studied under the guise of temporally split operators [10]. In either case, the operator renormalization is changed by an amount which comes from the self-energy. We will not consider this generalization further. 
The most general heavy-light bilinear in the full continuum theory is,

$$
J(x)=\bar{b}(x) \boldsymbol{\Gamma} q(x)
$$

Here $\boldsymbol{\Gamma}$ is any Dirac matrix, and $q$ is the light quark field. In the heavy quark effective theory, the corresponding operator (3.3) is [9],

$$
b^{\dagger}(x)\left(\begin{array}{ll}
\mathbf{1} & \mathbf{0}
\end{array}\right) \boldsymbol{\Gamma} q(x) .
$$

In a Dirac basis, the two-by-four matrix preceding $\boldsymbol{\Gamma}$ takes the form $\left(\begin{array}{ll}\mathbf{1} & \mathbf{0}\end{array}\right)$ and projects onto the upper two rows of $\boldsymbol{\Gamma}$. Applying the above recipe to the local operator $b^{\dagger}(n)\left(\begin{array}{ll}\mathbf{1} & \mathbf{0}\end{array}\right) \boldsymbol{\Gamma} \mathbf{q}(\mathbf{n})$ we are led to consider,

$$
b^{\dagger}(n)(\mathbf{1} \mathbf{0}) \boldsymbol{\Gamma} q(n)-\frac{r}{2} b^{\dagger}(n)(\mathbf{1} \mathbf{0}) \boldsymbol{\Gamma} \sum_{\mu} \gamma_{\mu}\left[U_{\mu}(x) q(n+\mu)-U_{\mu}(n-\hat{\mu})^{\dagger} q(n-\hat{\mu})\right] .
$$

The first term in this expression is the usual local current used to determine hadronic matrix elements. The calculations necessary to renormalize this part of the current were mostly done in previous papers [11][13], however it receives additional contributions coming from the new term in the action (3.1). The remainder of the current, which is naively order $a$, will also affect the renormalization of the current when it appears in loop diagrams with loop momenta of order $a^{-1}$. We now turn to the perturbative renormalization of the improved heavy-light current with the improved Wilson action of Sheikholeslami and Wohlert.

\section{Renormalization of the Improved Current}

The lattice renormalization of the heavy-light current (3.5) gives the ratio of the lattice operator to its counterpart in the continuum theory. We can divide the diagrams that contribute to this ratio into two parts. Those that give heavy and light quark wave function renormalization, and the 1PI vertex correction diagrams.

The difference between wave function renormalization of the heavy quark on the lattice and in the continuum is $g^{2} /\left(12 \pi^{2}\right)$ times a constant $e=4.53$ computed in references [11] and [13]. ${ }^{\dagger}$ The corresponding light quark wave function renormalization constant was calculated in [5]. The results for the self energy-graphs which

$\dagger$ Here we are using the reduced value of $e$ which is appropriate if one fits correlation functions containing the propagator (2.4) to $A \mathrm{e}^{-B\left(n_{0}-m_{0}\right) a}$ [10][11][13]. 


\begin{tabular}{|c|c|c|c|}
\hline$r$ & $d_{1}$ & $d_{2}$ & $\Delta_{\Sigma_{1}}$ \\
\hline 1.00 & 5.46 & -7.22 & -9.21 \\
\hline 0.75 & 5.76 & -7.23 & -8.62 \\
\hline 0.50 & 6.30 & -7.00 & -7.80 \\
\hline 0.25 & 7.37 & -5.72 & -6.73 \\
\hline 0.00 & 8.79 & 0.00 & -6.04 \\
\hline
\end{tabular}

Table 1. Previously Computed r-dependent Quantities [5][11][13].

we will need are given in terms of $\Delta_{\Sigma_{1}}$, defined in reference [5] (when consulting their expressions for $\Delta_{\Sigma_{1}}$, note that we have taken $F_{0001}=1.31$ [14]).

We now turn to the vertex correction diagrams of figures 1 and 2 . The techniques we use to evaluate these diagrams have been discussed in detail in a number of references [10][11], and will not be reviewed here.

At zero external momentum, 1(a) vanishes. As noted below equation (3.5), the operator has two pieces: one which is the local unimproved operator, and an additional piece coming from improvement. Thus the contribution of 1 (b) can be split up into two parts which we will call the "unimproved" and "improved" contributions here and in the following paragraph. The "unimproved" contribution is $[11][13]$,

$$
\frac{g^{2}}{12 \pi^{2}}\left(d_{1}+G d_{2}\right)
$$

We have defined the $c$-number $G$ by $G \boldsymbol{\Gamma}=\gamma_{0} \boldsymbol{\Gamma} \gamma_{0}$. The analytical expressions for $d_{1}$ and $d_{2}$ can be found in reference [11]. We tabulate the constant $\Delta_{\Sigma_{1}}$ and the constants $d_{1}$ and $d_{2}$ in Table 1 for several values of the Wilson mass parameter $r$. Errors in Table 1 and 2 are at most $\mathcal{O}(1)$ in the last decimal place.

The "improved" contribution from diagram 1(b) can be combined with the contribution from the other three diagrams in figure 1 . In order to compactly quote the analytic expressions for this result, we use the notation of references [15] and [16] for the following commonly occurring combinations:

$$
\begin{aligned}
& \Delta_{1}=\sum_{\mu} \sin ^{2} \frac{l_{\mu}}{2}, \quad \Delta_{4}=\sum_{\mu} \sin ^{2} l_{\mu} \\
& \Delta_{2}=\Delta_{4}+4 r^{2} \Delta_{1}^{2}, \quad \Delta_{5}=\sum_{\mu} \sin ^{2} \frac{l_{\mu}}{2} \sin ^{2} l_{\mu} .
\end{aligned}
$$




\begin{tabular}{|c|c|c|c|c|}
\hline$r$ & $\Delta d_{1}$ & $\Delta d_{2}$ & $\Delta d_{1}^{\prime}$ & $\Delta d_{2}^{\prime}$ \\
\hline 1.00 & -6.64 & -5.84 & 3.43 & 6.16 \\
\hline 0.75 & -5.30 & -3.75 & 2.53 & 4.94 \\
\hline 0.50 & -3.61 & -1.87 & 1.55 & 3.66 \\
\hline 0.25 & -1.52 & -0.45 & 0.55 & 2.15 \\
\hline 0.00 & 0.00 & 0.00 & 0.00 & 0.00 \\
\hline
\end{tabular}

Table 2. Changes to $d_{1}$ and $d_{2}$ as a function of $r$.

Additional combinations, $\Delta_{1}^{(3)}, \Delta_{2}^{(3)}$, and $\Delta_{4}^{(3)}$, are the same as above except the sums on $\mu$ run only from 1 to 3 . Given these definitions the total "improved" contribution from figure 1 to the vertex renormalization is,

$$
\frac{g^{2}}{12 \pi^{2}}\left(\Delta d_{1}+G \Delta d_{2}\right)
$$

where,

$$
\Delta d_{1}=\frac{-3 r^{2}}{16} \int \frac{d^{4} l}{\pi^{2}} \frac{4-\Delta_{1}}{\Delta_{2}}, \quad \Delta d_{2}=\frac{-r^{3}}{2} \int \frac{d^{3} l}{\pi} \frac{\Delta_{1}^{(3)}}{\Delta_{2}^{(3)}}
$$

We now turn to the contributions depicted in figure 2 which are due to the additional term in the action (3.1). In these figures, the insertion of this term is denoted with a cross. A tadpole diagram which vanishes has not been depicted. The result for the two diagrams depicted is,

$$
\frac{g^{2}}{12 \pi^{2}}\left(\Delta d_{1}^{\prime}+G \Delta d_{2}^{\prime}\right)
$$

where,

$$
\Delta d_{1}^{\prime}=\frac{r^{2}}{4} \int \frac{d^{4} l}{\pi^{2}} \frac{\Delta_{4}\left(3-\Delta_{1}\right)+\Delta_{5}}{4 \Delta_{1} \Delta_{2}}, \quad \Delta d_{2}^{\prime}=\frac{r}{2} \int \frac{d^{3} l}{\pi} \frac{\Delta_{4}^{(3)}\left(1+r^{2} \Delta_{1}^{(3)}\right)}{4 \Delta_{1}^{(3)} \Delta_{2}^{(3)}} .
$$

Combining the various results in this section, we find that the ratio of the lattice to continuum operators is,

$$
1+\frac{g^{2}}{12 \pi^{2}}\left[\left(d_{1}+\Delta d_{1}+\Delta d_{1}^{\prime}\right)+\left(d_{2}+\Delta d_{2}+\Delta d_{2}^{\prime}\right) G+\frac{1}{2} e-\frac{1}{2} \Delta_{\Sigma_{1}}-1\right] .
$$

The dependance on $\mu a$ has been eliminated by setting $\mu=1 / a$. 


\section{Conclusions}

We illustrate the use of the results of the previous section for the case of most interest, the current which determines the $B$ meson decay constant, $f_{B}$. In that case, for reasonable values of the input parameters, the ratio of the continuum effective theory to full theory bilinears is numerically 0.98 [9][13]. We need to multiply this by the ratio given in eq. (4.7). For $\boldsymbol{\Gamma}=\gamma_{0} \gamma_{5}$, the constant $G$ (which appears in (4.7)) is -1 . We take $g^{2}=1.8$ which is appropriate for effects arising from the scale $\pi / a$ with $1 / a=2 \mathrm{GeV}$ as argued in [17]. Taking values from tables 1 and 2 with $r=1.00$, equation (4.7) gives 1.23 and the product of the two ratios is 1.20 (as compared to 1.28 in the unimproved case). To obtain the physical value of $f_{B}$, one divides the lattice results for the improved current by this number.

The operator we have renormalized is corrected both to order $g^{2}$ and to order $a$. As noted in the introduction both of these corrections are thought to be in the 20 to $30 \%$ range. Analytically, the next perturbative corrections are proportional to $g^{2} a, g^{4}$, or $a^{2}$ times powers of $g^{2} \ln a$ [3], and there is numerical evidence that these further corrections are at the few per cent level for currents made from two Wilson fermion fields [3]. Thus it is hoped that the improved currents renormalized here will lead to a considerably more precise lattice determination of $f_{B}$ and other heavy quark matrix elements.

Note Added. While preparing this manuscript we learned of unpublished work on this subject [18][19] referenced in a paper on lattice measurements of several quantities using an improved action [20]. Martinelli and Rossi [18] argue that it is unnecessary to change the heavy quark action up to order $a^{2}$. This is consistent with our conclusion in section two that the heavy quark action is not in need of improvement at any order in $a$. The result of Borrelli and Pittori [19] cited in reference [20] is consistent with our factor above if one takes $g^{2}=1.0$ rather than 1.8 .

\section{Acknowledgements}

OFH was supported in part by the National Science and Engineering Research Council of Canada, and les Fonds FCAR du Québec. BRH was supported in part by the Department of Energy under Contract No. DE-AT03-88ER 40383 Mod A006Task C. 


\section{References}

[1] K. Symanzik, Nucl. Phys. B 226 (1983) 187.

[2] M. Lüscher and P. Weisz, Commun. Math. Phys. 97 (1985) 59.

[3] E. Gabrielli et al, in Lattice 90, ed. by U. M. Heller et al, Nucl. Phys. B (Proc. Suppl.) 20 (1991) 448;

G. Heatlie et al, Nucl. Phys. B352 (1991) 266.

[4] H. W. Hamber and C. M. Wu, Phys. Lett. 133B (1983) 351; Phys. Lett. 136B (1984) 255.

[5] E. Gabrielli et al, Nucl. Phys. B362 (1991) 475.

[6] B. Sheikholeslami and R. Wohlert, Nucl. Phys. B259 (1985) 572.

[7] E. Eichten, in Field Theory on the Lattice, edited by A. Billoire et al., Nucl. Phys. B (Proc. Suppl.) 4 (1988) 170.

[8] G. P. Lepage and B. A. Thacker, ibid, p. 199.

[9] W. E. Caswell and G. P. Lepage, Phys. Lett. 167B (1986) 437;

H.D. Politzer and M. B. Wise, Phys. Lett. B 208 (1988) 504;

E. Eichten and B. Hill, Phys. Lett. B 234 (1990) 511;

B. Grinstein, Nucl. Phys. B339 (1990) 253;

H. Georgi, Phys. Lett. B 240 (1990) 447;

M. J. Dugan, M. Golden, and B. Grinstein, HUTP/91-A045, to appear in Phys. Lett. B.

[10] O. F. Hernández and B. R. Hill, UCLA/91/TEP/51, to appear in Phys. Lett. B.

[11] E. Eichten and B. Hill, Phys. Lett. B 240 (1990) 193.

[12] J. M. Cornwall and G. Tiktopoulos, Phys. Rev. D 15 (1977) 2937;

E. Eichten and F. Feinberg, Phys. Rev. D 23 (1981) 2724.

[13] Ph. Boucaud, C. L. Lin, and O. Pène, Phys. Rev. D 40 (1989) 1529; Phys. Rev. D 41 (1990) 3541(E).

[14] A. Gonzales-Arroyo and C. P. Korthals-Altes, Nucl. Phys. B205 (1983) 46.

[15] G. Martinelli and Y.-C. Zhang, Phys. Lett. 123B (1983) 433.

[16] J. M. Flynn, O. F. Hernández, and B. R. Hill, Phys. Rev. D 43 (1991) 3709.

[17] G. P. Lepage and P. B. Mackenzie, in Lattice 90, ed. by U. M. Heller et al, Nucl. Phys. B (Proc. Suppl.) 20 (1991) 173.

[18] G. Martinelli and G. C. Rossi, unpublished.

[19] A. Borrelli and C. Pittori, unpublished.

[20] G. Martinelli, C. T. Sachrajda, G. Salina, and A. Vladikas, SHEP 91/92-6. 


\section{Figure Captions}

Fig. 1: The vertex correction diagrams resulting from improving the light quark operator

Fig. 2: The vertex correction diagrams resulting from improving the action 